\title{
Feature Extraction of EEG Signal using Wavelet Transform
}

\author{
Ashwini Nakate \\ P.G.Student \\ Department of Electronics \& \\ Telecommunication Engineering \\ N.K.O.C.E. Solapur
}

\author{
P.D. Bahirgonde \\ Associate Professor \\ Department of Electronics \& \\ Telecommunication Engineering \\ N.K.O.C.E. Solapur
}

\begin{abstract}
EEG signal analysis is such an important thing for disease analysis and brain-computer analysis. Using Electroencephalography (EEG) monitoring the state of the user's brain functioning and treatment for any psychological disorder, where the difficulty in learning and comprehending the arithmetic exists and it could allow for analysis disease the user to train the corresponding brain. In this paper, we proposed a method for EEG signal processing includes signal de-noising, segmentation of de-noise signal using PCM and signal segments feature extraction done using wavelet as an alternative to the commonly used discrete Fourier transform (DFT).These feature classified using support vector machine classifier, Using the Matlab software proposed method accompanied.
\end{abstract}

\section{Keywords}

EEG (Electroencephalography), segmentation, PCM, DWT SVM.

\section{INTRODUCTION}

For brain signal acquisition various methods used such as electroencephalography (EEG), Functional Magnetic Resonance Imaging (FMRI), Near Infra-Red Spectroscopy (NIRS) and Magneto encephalography (MEG). From this method EEG is wielding used signal acquisition method because of high temporal resolution and safe for use [1]. EEG used in medical purpose and also for brain computer interfacing (BCI).That EEG signal processing important for proper analysis disease. Signal processing of EEG is fundamental for analysis of brain activity and diagnosis of normality or abnormality of signal that is important for analysis of any disease. In this paper devote on EEG signal processing that followed by signal de-noising, segmentation of de-noise signal using "Principal Component Analysis (PCA)" it forms the feature vector. The paper devoted on EEG signal processing, follow by below graph.

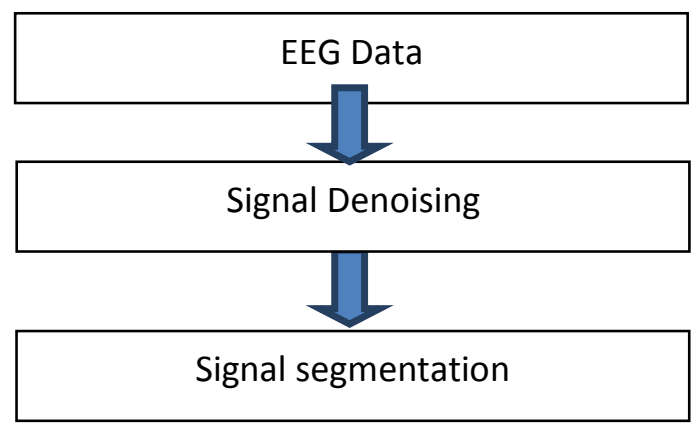

\section{SIGNAL DE-NOISING}

During EEG recording many of other influence introduce noise which called as artifact. These artifacts come from patient body or instrument, as an example eyes movement, the heart, muscles and line power. Before processing EEG, removal of this artifact is primary task and it form fundamental step for EEG signal processing.

For signal processing of EEG data set taken from online available PhysioNet.

(http://physionet.org/physiobank/database/chbmit)

EEG signal de-noising can be done by both wavelet and time domain method. Here we used Lowpass filter for de-noising. Most of time EEG signal contain neural information below $100 \mathrm{~Hz}$ so it's beneficial to use low pass filter.

Low pass filter implementation carried thrown matlab which will allow removing noise and artifact from the original signal extracted from patient [2]. Using filter design and process, Lowpass filter is implemented as shown below figure.

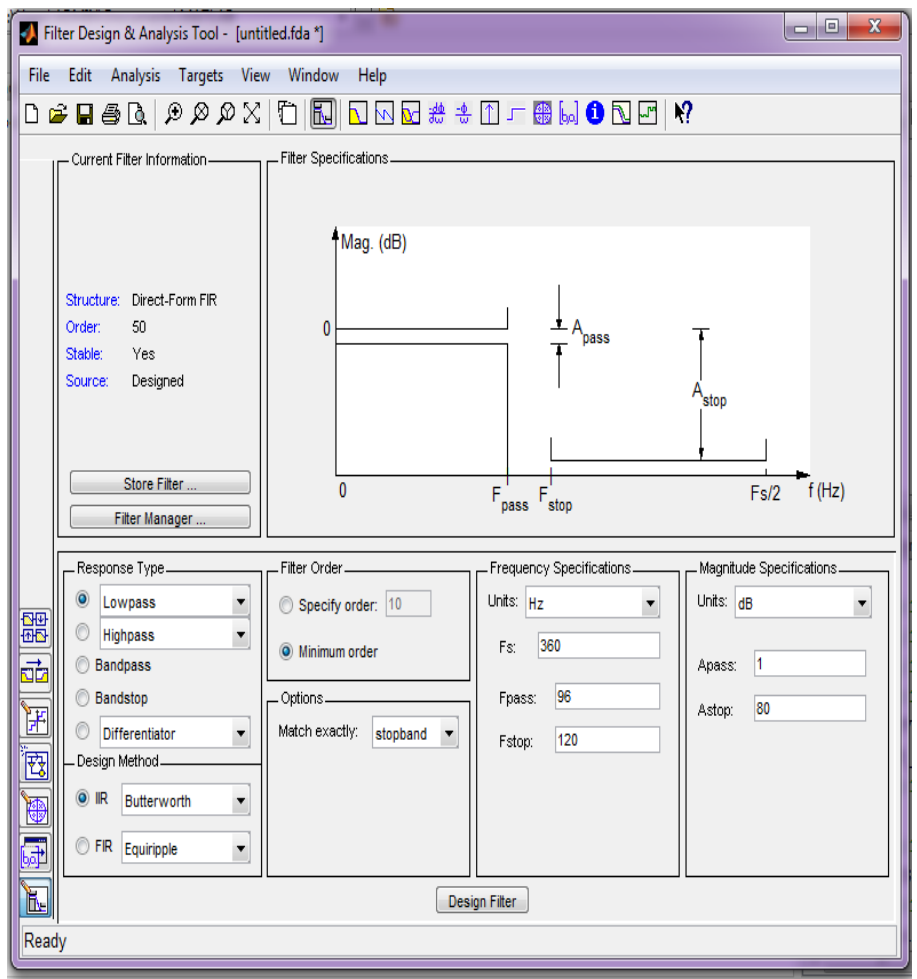

Fig. 1: Low pass filter implementation 
After design filter implemented filter apply to EEG signal, then result shown as follows.

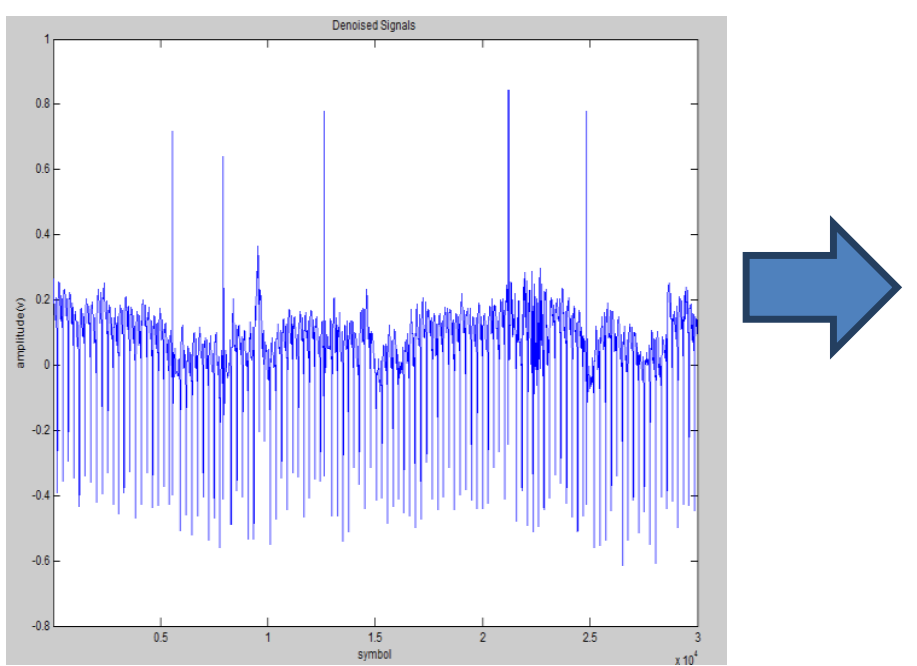

Fig. 2: Original signal

\section{SIGNAL SEGMENTATION USING PCM}

Segmentation of EEG signals should be performed over all channels. In most cases it is important to extract information present in all parallel time series at first. The principal component analysis good for this type requirement and large data set, it took a large set of data and identified an optimal new basis in which to re-express the data [3].

The first task of PCA is to identify a new set of orthogonal coordinate axes through the data. This is achieved by finding the direction of maximal variance through the coordinates in the signal. It is equivalent to obtaining the (least-squares) line of best fit through the plotted data. We call this new axis the first principal component of the data. Figure4. Show first principal component of the signal.

Assume that we start with a data set that is represented in terms of an $\mathrm{m} \times \mathrm{n}$ matrix, $\mathrm{X}$ where the $\mathrm{n}$ columns are the samples (e.g. observations) and the $\mathrm{m}$ rows are the variables. We wish to linearly transform this matrix, $X$ into another matrix, $Y$, also of dimension $\mathrm{m} \times \mathrm{n}$, so that for some $\mathrm{m} \times \mathrm{m}$ matrix, $\mathrm{P}$,

$$
Y=P X
$$

This equation represents a change of basis. If we consider the row vector $\mathrm{P}$ to be the row vectors $\mathrm{p} 1, \mathrm{p} 2, \ldots, \mathrm{pm}$, and the columns of $\mathrm{X}$ to be the column vectors $\mathrm{x} 1, \mathrm{x} 2, \ldots, \mathrm{xn}$, then can be interpreted in the following way.



Fig. 3: De-noise signal

$$
\begin{aligned}
& \mathrm{PX}=\left(\mathrm{P} x_{1} \mathrm{P} x_{2} \ldots \mathrm{Pxn}\right) \\
& =\left[\begin{array}{c}
p 1 . x 1, p 1 . x 2 \ldots \ldots . . p 1 . x n \\
p 2 . x 1, p 2 . x 2 \ldots \ldots . . p 2 . x n \\
:::: \\
p m . x 1, p m . x 2 \ldots \ldots . . .: \text { pm. } x n
\end{array}\right]
\end{aligned}
$$

Equation represents a change of basis and thus can have many interpretations.

$\mathrm{P}$ is a matrix that transforms $\mathrm{X}$ into $\mathrm{Y}$. Geometrically $\mathrm{P}$ is a rotation and a stretch which again transforms $\mathrm{X}$ into $\mathrm{Y}$. The rows of $\mathrm{P},\{\mathrm{p} 1 \ldots \mathrm{pm}\}$, are a set of new basis vectors for expressing the columns of $\mathrm{X}$.

Note that $\mathrm{pi}, \mathrm{x}_{\mathrm{i}}$ implies from $\mathrm{R}_{\mathrm{Ym}}$, and so $\mathrm{p}_{\mathrm{i}} \cdot \mathrm{x}_{\mathrm{i}}$ is just the standard Euclidean inner (dot) product. This tells us that the original data, $\mathrm{X}$ is being projected onto the columns of $\mathrm{P}$. Thus, the rows of $\mathrm{P},\{\mathrm{p} 1, \mathrm{p} 2 . \mathrm{pm}\}$ are a new basis for representing the columns of $\mathrm{X}$. figure 5 shows signal plot using new basic function.

The rows of $\mathrm{P}$ will later become our principal component directions [4], [5]. Principal component analysis defines independence by considering the variance of the data in the original basis. It forms a feature vector for further signal processing. 




Fig. 4: First principal component

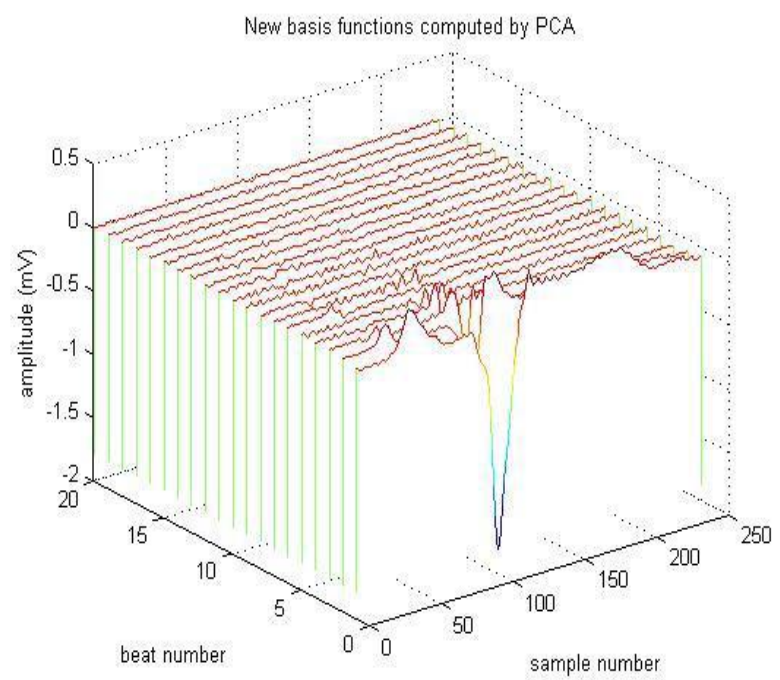

Fig. 5: New basic function by PCM

This information can be summarized in a plot of the variances (nonzero eigenvalues) with respect to the principal component number (eigenvector number), which is given in Figure 6, below.

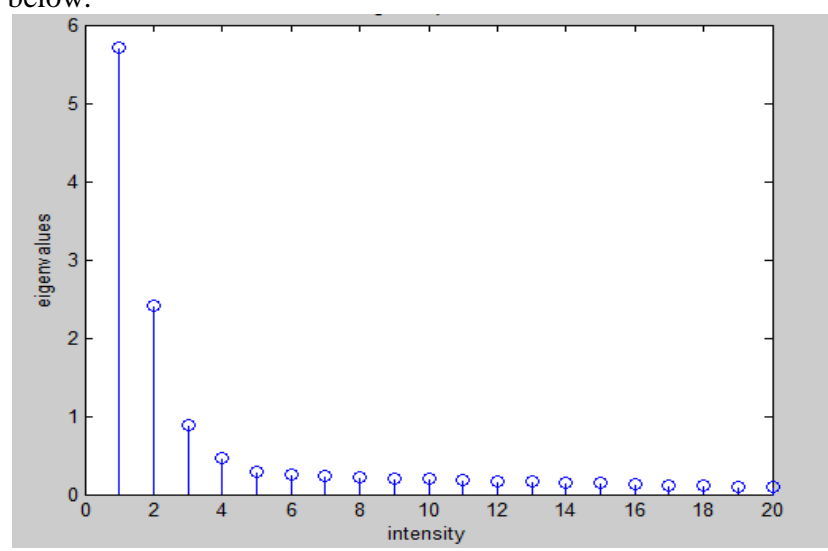

Fig. 6: Eigen spectrum

\section{FEATURE EXTRACTION}

In pattern recognition, feature extraction is a special form of dimensionality reduction. When the input data to an algorithm is too large to be processed and it is suspected to be notoriously redundant (much data, but not much information) then the input data will be transformed into a reduced representation set of features (also named features vector). Transforming the input data into the set of features is called feature extraction. If the features extracted are carefully chosen it is expected that the features set will extract the relevant information from the input data in order to perform the desired task using this reduced representation instead of the full size input. The following study is devoted to the wavelet domain signal feature extraction and comparison of results achieved [6], [7].

The frequency content of EEG signal provides useful information than time domain representation. The wavelet transform gives us multi-resolution description of a nonstationary signal. EEG is non-stationary signal hence wavelet is suited for EEG signals [1]. At high frequencies it represents a good time resolution and for low frequencies it represents better frequency resolution. This multi-scale feature of the Wavelet allows the decomposition of a signal into a number of scales, each scale representing a particular coarseness of the signal under study. The procedure of multiresolution decomposition of a signal $\mathrm{x}[\mathrm{n}]$ is schematically shown in Fig.7

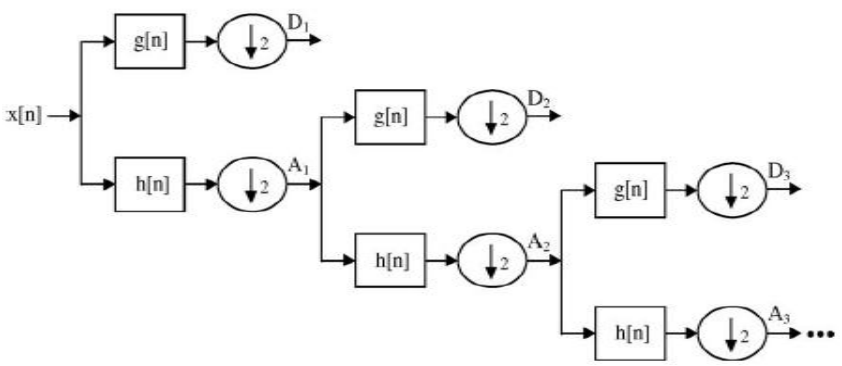

Fig. 7: Sub band decomposition of discrete wavelet transforms implementation; $\mathrm{g}[\mathrm{n}]$ is the high-pass filter, $h[n]$ is the low-pass filter

The wavelet coefficients were computed using daubechies wavelet of order 2 because its smoothing features are more suitable to detect changes in EEG signal. In the present study, the EEG signals were decomposed into details D2.

The extracted wavelet coefficients provide a compact representation that shows the energy distribution of the EEG signal in time and frequency band. Therefore, the computed detail and approximation wavelet coefficients of the EEG signals were used as the feature vectors representing the signals. There are no. of wavelet coefficients. In order to reduce the dimensionality of the feature vectors, statistics over the set of the wavelet coefficients were used. The following statistical features were used to represent the time-frequency distribution of the

EEG signals:

-Mean of the wavelet coefficients in each sub band.

-Standard deviation of the wavelet coefficients in each sub band. 

signal segments analysis

\begin{tabular}{|l|l|l|l|l|l|l|}
\hline Feature source & \multicolumn{3}{|l|}{ Energy } & & \multicolumn{3}{l|}{ Mean } \\
& \multicolumn{1}{l|l|}{$S_{1}$} & $S_{2}$ & $S_{3}$ & $S_{1}$ & $S_{2}$ & $S_{3}$ \\
\hline DWT & 10.37 & 14.68 & 10.31 & 0.0091 & 0.006 & 0.001 \\
\hline DFT & 0.0001 & 0.002 & 0.000 & 0.0002 & 0.003 & 0.002 \\
\hline
\end{tabular}

\section{CLASSIFICATION}

In the context of biomedical signal processing, especially with application to EEG signals, the classification of the data in feature spaces is often required. The objective of classification is to draw a boundary between two or more classes and to label them based on their measured features. In a multidimensional feature space this boundary takes the form of a separating hyperplane. The art of the work here is to find the best hyperplane that has a maximum distance from all the classes. There have been several clustering and classification techniques developed within the last forty years. Among them artificial neural network (ANN), hidden Markov modeling, fuzzy logic and support vector machine are popular.

\section{Support Vector Machines}

The SVM proposed by Vapnik [9] has been studied extensively for classification, regression, and density estimation. Fig. 3 shows the architecture of the SVM. SVM maps the input patterns into a higher dimensional feature space through some nonlinear mapping chosen a priori. A linear decision surface is then constructed in this highdimensional-feature space. Thus, SVM is a linear classifier in the parameter space, but it becomes a nonlinear classifier as a result of the nonlinear mapping of the space of the input patterns into the high-dimensional feature space.

$$
\operatorname{margin} \equiv \underset{\mathbf{x} \in D}{\arg \min } d(\mathbf{x})=\underset{\mathbf{x} \in D}{\arg \min } \frac{|\mathbf{x} \cdot \mathbf{w}+b|}{\sqrt{\sum_{i=1}^{d} w_{i}^{2}}}
$$

The above illustration is the maximum linear classifier with the maximum range. In this context it is an example of a simple linear SVM classifier. The goals of SVM are separating the data with hyper plane and extend this to nonlinear boundaries using kernel trick [10]. The Kenral trick allows SVM's to form nonlinear boundaries. Steps involved in kernel trick are given below.

[a] The algorithm is expressed using only the inner products of data sets. This is also called as dual problem.

[b] Original data are passed through non linear maps to form new data with respect to new dimensions by adding a pair wise product of some of the original data dimension to each data vector.

[c] Rather than an inner product on these new, larger vectors, and store in tables and later do a table lookup, we can represent a dot product of the data after doing non linear mapping on them. This function is the kernel function.
Some of the popular Kernel functions are as follows [10];

a) Radial Kernel Function (RBF)

b) Linear Kernel Function

c) Polynomial Kernel Function

d) Gaussian Function

\section{CONCLUSIONS}

Feature extraction was done using discrete wavelet transform. EEG signals were decomposed up to level four using daubechies wavelet of order 2. Wavelet coefficients were computed. Mean, standard deviations of the wavelet coefficient were estimated. When this parameter compared with Fourier transform, wavelet give best result. Also Fourier transform is not suitable to EEG signal due to its nonstationary nature.

\section{REFERENCES}

[1] M. Rajya Lakshmi, Dr. T. V. Prasad, Dr. V. Chandra Prakash "Survey on EEG Signal Processing Methods" International Journal of Advanced Research in Computer Science and Software Engineering Volume 4, Issue 1, January 2014.

[2] Miguel Rivera, Laura Salas "Monitoring of Micro-sleep and Sleepiness for the Drivers Using EEG Signal" School of Innovation, Design and Engineering (IDT) Malardalen University Vasteras, Sweden.

[3] Jon Shlens "A Tutorial on Principal Component Analysis (Derivation, Discussion and Singular Value Decomposition)" 25 March 2003

[4] Mark Richardson "Principal Component Analysis" May 2009

[5] Jon Shlens "A TUTORIAL ON PRINCIPAL COMPONENT ANALYSIS"

[6] Dipti Upadhyay "Classification of EEG Signals under Different Mental Tasks Using Wavelet Transform and Neural Network with One Step Secant Algorithm" International Journal of Scientific Engineering and Technology, Volume 2 Issue 4, pp : 256-259

[7] Pravin A. Kharat, Sanjay V. Dudul "Daubechies Wavelet Neural Network Classifier for the Diagnosis of Epilepsy" ,wseas transactions on biology and biomedicine.

[8] Nandish.M, Stafford Michahial, Hemanth Kumar P, Faizan Ahmed "Feature Extraction and Classification of EEG Signal Using Neural Network Based Techniques", International Journal of Engineering and Innovative Technology (IJEIT) Volume 2, Issue 4, October 2012

[9] P Bhuvaneswari, J Satheesh Kumar "Support Vector Machine Technique for EEG Signals" International Journal of Computer Applications (0975 - 8887) Volume 63- No.13, February 2013

[10] Vikramaditya Jakkula "Tutorial on Support Vector Machine (SVM)" School of EECS, Washington State University, Pullman 99164. 